\title{
Honeybees, Bumblebees and Leaf-cutter Bees Vary in the Effect of a Simulated Pathogen Challenge on Individual Thermoregulation
}

Victoria Blanchard ( $\square$ viki.blanchard.2018@live.rhul.ac.uk)

Royal Holloway University of London

James E. Cresswell

University of Exeter

David R Tarpy

North Carolina State University

\section{Research Article}

Keywords: Lipopolysaccharide, Thermoregulation, Apis, Bombus, Megachile

Posted Date: June 17th, 2021

DOl: https://doi.org/10.21203/rs.3.rs-613610/v1

License: (c) (i) This work is licensed under a Creative Commons Attribution 4.0 International License.

Read Full License 


\section{Abstract}

Bees regulate their individual body temperatures by non-flight thermogenesis (NFT). The effects of a pathogen challenge on thermoregulation in bees generally is unknown, although honeybees have displayed opposing responses between two studies. To establish whether bees in general experience disruption of thermoregulation under pathogen challenge, we investigated a representative species of each of three major bee social backgrounds (honeybees, Apis mellifera; bumblebees, Bombus impatiens, and solitary bees, Megachile rotundata) and measured the body surface temperatures of individual bees as they recovered from cold torpor by NFT after injection with lipopolysaccharide (LPS) solution, which simulated a pathogen challenge. We found that LPS injection affected rewarming in the annually eusocial $B$. impatiens, but not $A$. mellifera or the solitary M. rotundata. Specifically, the pathogen challenge increased post-recovery body temperatures by $2{ }^{\circ} \mathrm{C}$ in $B$. impatiens individuals. Our findings indicate that immune responses by individual bees can interfere with thermoregulation, but this effect is not consistent among major bee species.

\section{Introduction}

Bees cannot fly in cold conditions without first warming their flight muscles ${ }^{1}$. Bees use non-flight thermogenesis (NFT) to raise their thoracic temperature to the flight threshold ${ }^{2}$. This allows them to forage for food, evade predation, and gather resources. Social species also use NFT to incubate their brood $^{3,4}$, which maximises the rate of colony growth. In general, effective thermoregulation by active NFT is a critical adaptation for bees.

External factors such as starvation can limit an individual's ability to thermoregulate effectively ${ }^{2,5}$. For example, exposure to pesticides can disrupt thermogenesis in bees ${ }^{6,7}$. Potentially, other stressors may interfere with thermoregulation, such as pathogen challenge.

Bees maintain various immune responses, which include both cellular responses (phagocytosis, encapsulation) and humeral responses (the prophenoloxidase cascade and antimicrobial effectors). The high cost of active immunocompetence ${ }^{8}$ may divert limited resources from thermogenic metabolism. Alternatively, bees may exhibit a fever response if this were adaptive in combatting the proliferation of infection. Indeed, various insect species have demonstrated a preference for warmer environments or generated higher temperatures in response to pathogen challenges ${ }^{9}$. However, the extent to which the induction of an immune response in bees interferes with the regulation of body temperature is unclear.

Here we investigated a representative of each major social group. Specifically, we compare the highly eusocial Apis mellifera (honeybee), the annually eusocial Bombus impatiens (bumblebee), and the solitary Megachile rotundata (alfalfa leafcutter bee/ALCB) to see if an artificial immune challenge affects individual warming, and if this response differs regarding social background.

\section{Methods}


We collected $\sim 50$ newly eclosed bees $(A$. mellifera) from four different colonies with no signs of disease at the NC State Apiculture Program's Honeybee Research Facility (Raleigh, NC). We caged batches of 30 marked bees in plastic pots along with unmarked sisters from the same source colony (total of 40-50 bees per cage) and maintained them at $31.5^{\circ} \mathrm{C}^{10}$. We provided the bees with ad libitum $50 \%$ sucrose solution in feeders for at least 7 days before treatment.

We acquired 15 B. impatiens colonies from BioBest LTD (Leamington, Ontario, Canada). We transferred 100 newly emerged individuals from healthy colonies (screened for Crithidia bombi) into $200 \times 250 \times 150$ $\mathrm{mm}$ ventilated petri dishes at room temperature $\left(\sim 22 \pm 4^{\circ} \mathrm{C}\right)$ for $7-14$ days before treatment. Individuals were given $50 \%$ and allowed to feed ad libitum.

We obtained alfalfa leafcutter bees ( $M$. rotundata) from 500 nesting cells in January 2017 from the USDA-ARS Pollinating Insects Research Unit (Logan, Utah). The cells were screened for disease using $x-$ ray photography and kept at $4^{\circ} \mathrm{C}$ prior to incubation ${ }^{11}$. Cells incubated at $28^{\circ} \mathrm{C}$ within a $200 \times 250 \times 150$ $\mathrm{mm}$ wooden box for 18 days $^{12}$. We transferred females to individual $100 \times 15 \mathrm{~mm}$ petri dishes with $50 \%$ sucrose solution provided ad libitum.

To test the physiological response to a non-lethal immune challenge, we injected experimental subjects with lipopolysaccharides (LPS) from Escherichia coli 0128:B12 ${ }^{13,14}$. We suspended $10 \mathrm{mg}$ of LPS powder (Sigma Aldrich: L2755-10MG) in $2 \mathrm{ml}$ of Ringer's solution (VWR: AAJ67524-AP) ${ }^{8}$ and then injected $5 \mu \mathrm{l}$ of this solution through the pleural membrane between the second and third tergites of each bee using a sterilised glass capillary tube that had been pulled to a fine point. We anesthetised all bees with carbon dioxide for approximately 30 seconds until inert prior to treatment.

Our experiment included two control groups, a no-injection group and a group injected with $5 \mu$ l of Ringers solution only to control for mechanical injury. All bees were randomly assigned to treatment groups and were between 7 and 13 days post-eclosion on the day of injection.

To establish whether the immune challenge affected rewarming, we moved each bee to a refrigerated (4 ${ }^{\circ} \mathrm{C}$ ) environment to induce cold torpor 24 hours after injections. We kept $A$. mellifera and $M$. rotundata at this temperature for 30 minutes and $B$. impatiens for 1 hour because of their larger body size. We then transferred each bee to a mesh cage on a Styrofoam pad at laboratory room temperature $\left(\sim 22 \pm 4^{\circ} \mathrm{C}\right)$. We measured the body surface temperature of each individual immediately after removal from the refrigerator, and every minute for 30 minutes using a thermal imaging camera (Testo 870-1 Thermal Imager, Testo Ltd., Alton, UK). We set the camera's emissivity scale to $0.97^{15}$ and the reflected temperature compensation to $20^{\circ} \mathrm{C}$. We measured thoracic temperature using the mean of three measurements in the intertegular region using Testo IRSoft v3.7 (Testo Ltd., Alton, UK) ${ }^{7}$. From this series, we calculated each bee's rate of warming and final stable temperature. We also recorded dead bees with the same set up to ensure we saw active warming. We removed individuals from the final dataset that did not recover from cold torpor. 
To ensure that the LPS injections had stimulated an immune response, we conducted a zone of antimicrobial activity assay. Immediately after each final image, we anesthetised the bees with carbon dioxide and extracted haemolymph ${ }^{16}$. We pooled the samples according to treatment group within a species, and stored the pooled samples at $-80^{\circ} \mathrm{C}$. We then prepared a Micrococcus leuteus lawn using aseptic technique on standard agar plates. We placed $5 \mu \mathrm{l}$ aliquots of haemolymph onto the lawn and incubated the plates overnight at $30^{\circ} \mathrm{C}$. There was sufficient haemolymph for two replications of $A$. mellifera and $B$. impatiens treatment groups but only one $M$. rotundata plate (because of their smaller body size). We photographed the resulting zones of clearance (i.e. antimicrobial activity) and used Image J software (1.51k Wayne Rasband, National Institutes of Health, USA) to quantify antimicrobial activity by the mean of three diameter measurements.

\section{Results}

In B. impatiens, we detected variation among treatments in post-recovery equilibrated thoracic temperature (one-way ANOVA: $F_{2,29}=3.303, \mathrm{P}=0.0105 ;$ Fig. 1 ). LPS-injected bees had a surface temperature approximately $2{ }^{\circ} \mathrm{C}$ higher than control bees and the Ringers-only group (Tukey test, LPS vs Control: $P=0.028$, LPS vs Ringers: $P=0.066$ ). There was no difference between the stable temperature of the two control groups (Tukey test: $\mathrm{P}=0.951$ ).

We found no significant variation among treatment groups in post-recovery equilibrated thoracic temperature of $A$. mellifera and M. rotundata (Fig. 1).

None of the three species tested exhibited a change in rate of warming with an immune challenge (Fig. 2), although the rate of warming for live bees was higher than the ambient warming of dead bees (Student's $t$-test, $\left.t_{352}=-4.823, \mathrm{P}<0.001\right)$, which demonstrates active warming.

The areas of antimicrobial activity varied between treatment groups across all three species (one-way ANOVA: $F_{2,29}=6.089, \mathrm{P}=0.036$ ). LPS-challenged haemolymph had a much larger zone of inhibition (ZOI) than the control groups (Tukey HSD P $=0.018$ ) but not a significantly different ZOI than Ringer's-injected individuals (Tukey HSD P $=0.055$ ) indicating that there may have been a small degree of microbial contamination of the injured control groups, although there was no significant variation between control and Ringer's-injected areas of antimicrobial activity (Tukey HSD P $=0.625$ ).

\section{Discussion}

Our results demonstrate that thermoregulation in bees can be disrupted by a pathogen challenge in some species. Specifically, we found that an LPS-injection caused an increase in post-recovery equilibrated thoracic temperature in bumblebees, but not in honeybees and ALCBs.

Bumblebees may benefit from raising their internal body temperature with infection. Many animals are known to raise the threshold of their thermal equilibrium in response to a pathogen challenge, often 
referred to as a 'fever.' Several studies across vertebrates and invertebrates suggest that an increase in a subject's body temperature of $1-3^{\circ} \mathrm{C}^{17,18}$ in response to infection can increase survivorship ${ }^{19}$. We note that our study does not demonstrate a benefit from the altered thermal equilibrium and we have not established wither the alteration is transient or lasting.

In contrast to bumblebees, neither honeybees nor ACLBs exhibited a disruption of their thermal equilibrium in response to either wounding or LPS-injection. Previous work has found that honeybee colonies generate an increased brood temperature in response to infections of microsporidian fungi (Ascosphaera apis) ${ }^{20}$. In the colony, honeybees infected with $N$. ceranae displayed a preference for warmer areas of the comb, which they interpreted as a behavioural fever. However, isolated individuals did not alter their thoracic temperature in response to infection ${ }^{5}$. On this evidence, we provisionally propose that honeybees respond to microbial challenge more strongly at the whole-colony level than as individual bees. However, further experiments are needed to clarify how the immunophysiology of honeybees relates to variation in the type of immunochallenge and the social context of the individual.

The honeybee explanation of social background affecting the resilience of the individual's thermal equilibrium under immunochallenge cannot extend to $M$. rotundata. As a solitary bee, it is impossible that social hygiene has replaced the individual's immune response. Overall, this inconsistency highlights the provisional and incomplete understanding of the adaptive value of resilience of thermogenic recovery among bees generally, irrespective of the impact of social lifestyle. Further research in wider range of solitary bee species is therefore needed to assess whether solitary species seek out warmer temperatures as is the case within the colony in honeybees ${ }^{5}$ or in the general environment as in other solitary species ${ }^{9}$.

In conclusion, we found that $B$. impatiens individuals experienced a disturbance to their thermal equilibration in response to immune stimulation, but $A$. mellifera and $M$. rotundata did not. Our findings are only partially explained in the context of levels of sociality and should therefore stimulate further investigation into pathogen disruption of thermoregulation, the evolution of sociality and the adaptive significance of individual immunity.

\section{Declarations}

\section{Acknowledgements}

Jennifer Keller, Dr. Theresa Pitts-Singer, Ellen Klomps, Dr. Becky Irwin, and Sara June Connon provided the bees for this experiment. Dr. Eric Miller, Dr. Rebecca Ayme-Hughes, and Erin Harrell guided the zone of inhibition assays. Special thanks to James Withrow, Joe Milone, and Erin McDermott for their support throughout.

\section{Data Availability}

The dataset generated and analysed during the current study are available in the figshare repository, https://figshare.com/articles/dataset/ThermoProjectDataFormatted_xlsx/14770530 


\section{Author Contributions}

All authors contributed to the conception, analysis, and write-up of this work. Victoria Blanchard completed the practical elements. The authors declare no competing interest.

\section{References}

1. Heinrich, B., Esch, H. (1994) "Thermoregulation in Bees" American Scientist 82(2):164-170

2. Heinrich, B. (1974) "Thermoregulation in Endothermic Insects" Science 185(4153):747-756

3. Jones, J.C., Oldroyd, B.P (2006) "Nest Thermoregulation in Social Insects" Advances in Insect Physiology 33:153-191

4. Kronenberg, F., Heller, H.C. (1982) "Colonial thermoregulation in honeybees (Apis mellifera)" Journal of Comparative Physiology 148(1): 65-76

5. Campbell, J., Kessler, B., Mayack, C., Naug, D. (2010) “Behavioural fever in infected honeybees: parasitic manipulation or coincidental benefit" Parasitology 137(10):1487-1491

6. Belzunces, L.P., Vandame, R., Gu, X.f. (2001) “Joint effects of pyrethroid insecticides and azole fungicides on honey-bee thermoregulation." Hazards of Pesticides to Bees 297-8

7. Potts, R., Clarke, R. M., Oldfield, S.E., Wood, L.K., Hempel de Ibarra, N., Cresswell, J.E. (2018) "The effect of dietary neonicotinoid pesticides on non-flight thermogenesis in worker bumblebees (Bombus terrestris)" Journal of Insect Physiology 104: 33-39

8. Moret, Y. and Schmid-Hempel, P. (2000) Survival for Immunity: The Price of Immune System Activation for Bumblebee Workers Science 290:1166-1168

9. Stahlschmidt, Z.R., Adamo, S.A. (2013) "Context dependency and generality of fever in insect" Naturwissenschaften 100(7):691-696

10. Human, H., et al. (2013) Miscellaneous standard methods for Apis mellifera research. In The COLOSSBEEBOOK, Volume I: standard methods for Apis mellifera research. Journal of Apicultural Research 52(4)

11. Pitts-Singer, T., James, R. (2009) Prewinter management affects Megachile rotundata (Hymenoptera: Megachilidae) prepupal physiology and adult emergence and survival Journal of Economic Entomology 102:1407-1416

12. Huntzinger, C.I., James, R.R., Bosch, J., Kemp, W.P. (2008) Fungicide Tests on Adult Leafcutting Bees (Hymenoptera: Megachilidae) Journal of Economic Entomology 101(4):1088-1094

13. Laughton, A., Siva-Jothy, M. A (2010) Standardised Protocol for Measuring Phenoloxidase and Prophenoloxidase in the honeybee Apis mellifera Apidologie 42:140-149

14. Appler, R., Frank, S., Tarpy, D. (2015) Within-colony variation in the immunocompetency of managed and feral honeybees (Apis mellifera L.) in different urban landscapes Insects 6(4):912-925

15. Stabentheiner, A., Schmaranzer, S. (1987) Thermographic determination of body temperature in honeybees and hornets: calibration and applications Thermology 2:563-572 
16. Huang, Z. (2012) Protocol: Bee Bleeding http://www.cyberbee.net/index.php/research/bleeding (21st August 2017)

17. Kluger, M.J. (1979) Fever, its biology, evolution, and function. Princeton University Press, Princeton

18. Casterlin, M.E., Reynolds, W.W. (1982) "The Pyrogenic Responses of Invertebrates" in "Milton A.S. (eds) Pyretics and Antipyretics. Handbook of Experimental Pharmacology (Continuation of Handbuch der experimentellen Pharmakologie)" 60 Springer, Berlin

19. Boorstein, S.M., Ewald, P.W. (1987) "Costs and benefits of behavioural fever in Melanoplus sanguinipes infected by Nosema acridophagus" Physiological Zoology 60(5):586-595

20. Starks, P.T., Blackie, C.A., Seeley, T.D. (2000) "Fever in honeybee colonies" Naturwissenschaften 87:229-231

\section{Figures}

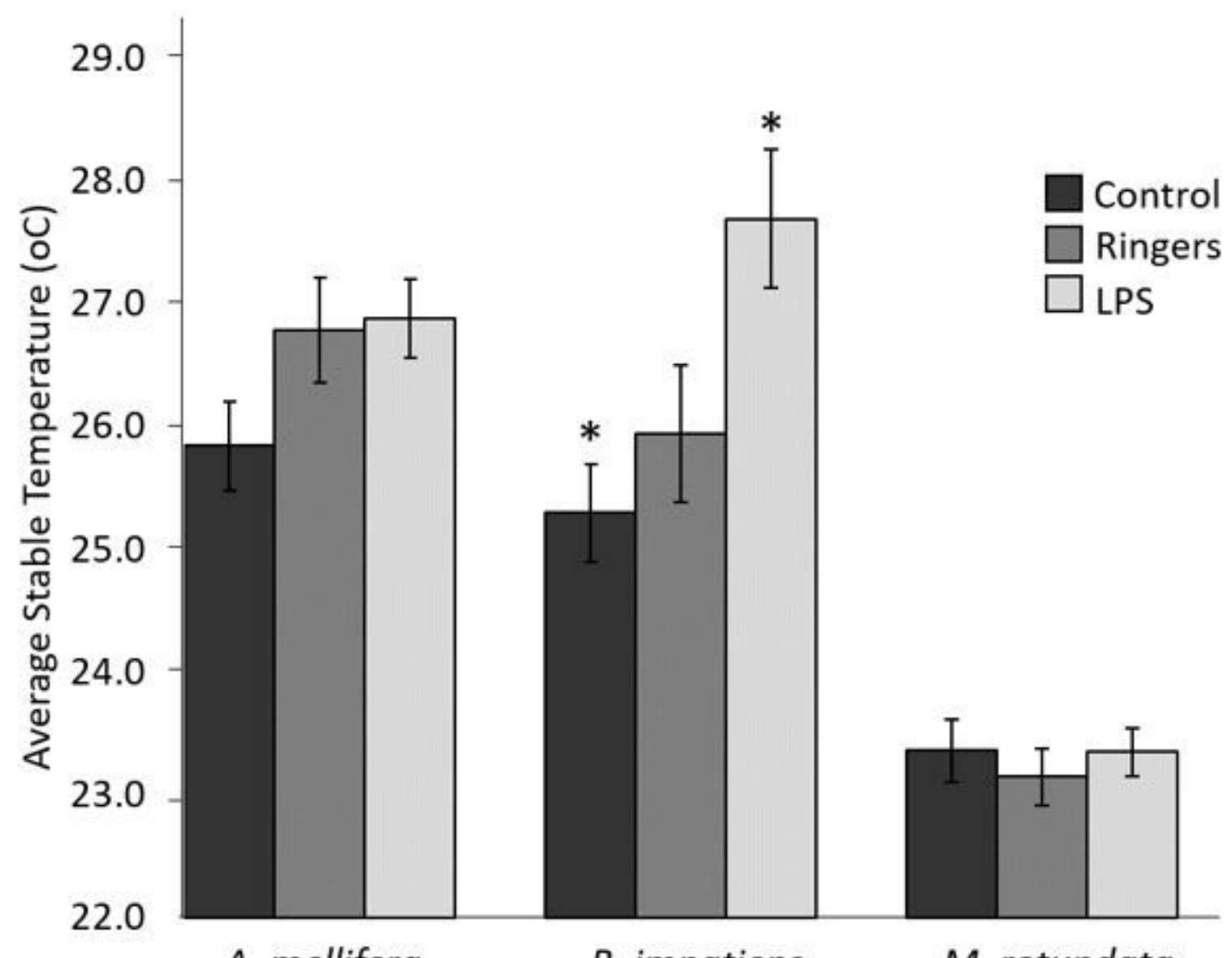

Figure 1 
Average stable thoracic temperature of A. mellifera, B. impatiens, and M. rotundata between 25 and 30 minutes post-rewarming. "Controls Combined" represents an average thoracic temperature of all Ringersinjected and non-injured bees. Treatments highlighted with an asterisk are significantly different from each other within a species at $p<0.05$

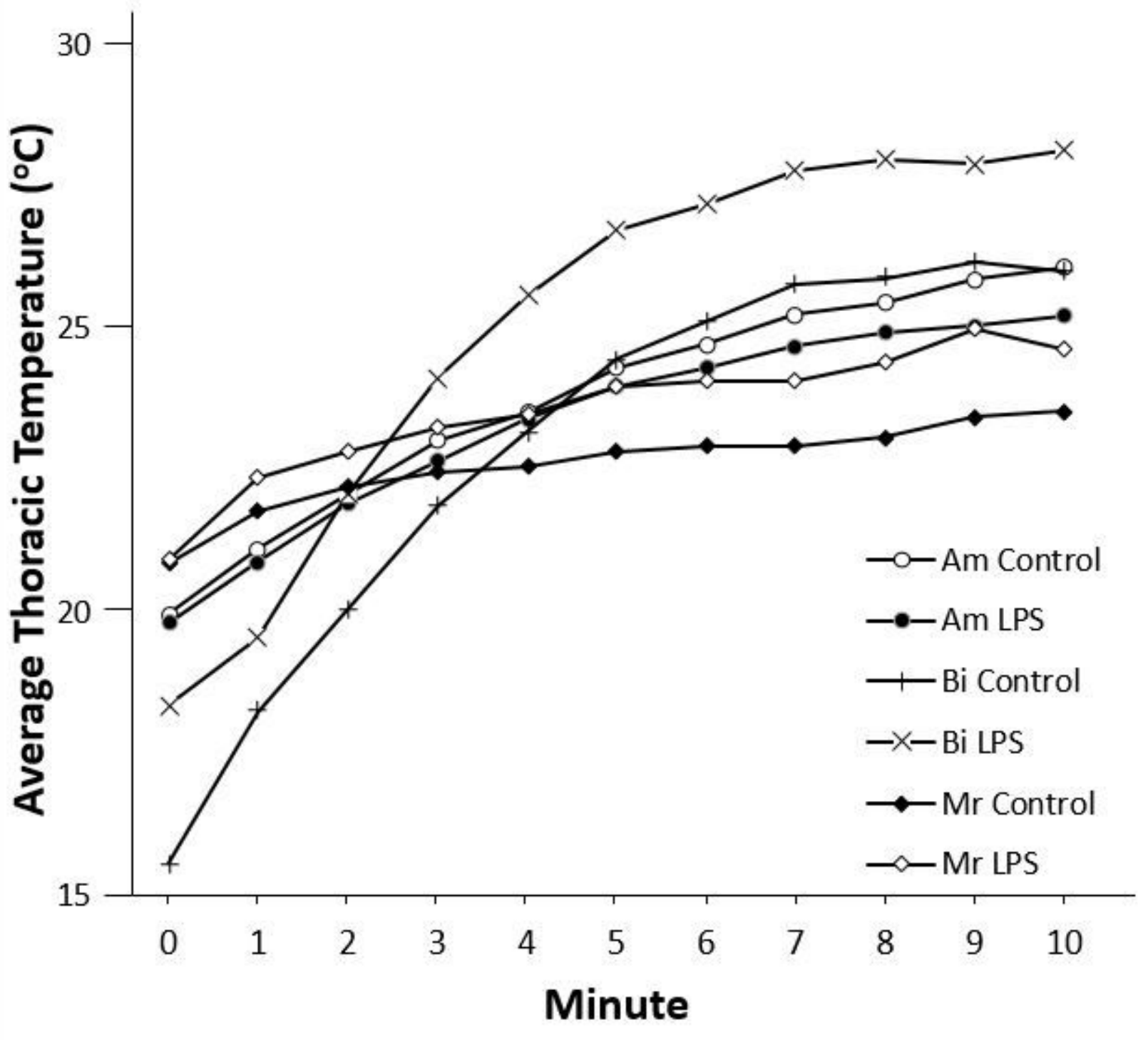

Figure 2

Warming curves of Apis mellifera (Am), Bombus impatiens (Bi), and Megachile rotundata (Mr) from cold torpor through the first 10 minutes of rewarming; after which point the temperatures are stable 